\title{
A Web System based on a Sports Injuries Model towards Global Athletes Monitoring
}

\author{
Patrícia Macedo ${ }^{1}$, Rui Neves Madeira ${ }^{1,3}$, André Correia ${ }^{1}$, Marco Jardim² \\ ${ }^{1}$ Escola Superior de Tecnologia de Setúbal and ${ }^{2}$ Escola Superior de Saúde, Instituto \\ Politécnico de Setúbal, Campus do IPS - Estefanilha, Setúbal, Portugal \\ ${ }^{3}$ CITI, Faculdade de Ciências e Tecnologia da Universidade Nova de Lisboa \\ 2829-516 Monte da Caparica - Portugal \\ \{patricia.macedo, rui.madeira,\}@estsetubal.ips.pt; marco.jardim@ess.ips.pt.
}

\begin{abstract}
This paper presents the project iReport SportsInjuries, which is a system with a focus on a Web application directed to sport health professionals, supporting the acquisition, analysis and dissemination of sports injuries information. This software will allow health professionals register and analyze sports injuries among sports populations. The application provides a reporting module that includes tables and charts to individually analyze injuries of a specific sports organization, a specific sport across organizations or a specific athlete, independently of where $\mathrm{s} / \mathrm{he}$ practices. The system also offers a module for a global analysis, which allows the iReport SportsInjuries to obtain the incidence and prevalence values, besides the socioeconomics costs, regarding sports injuries at a national level. In order to offer these features, it is based on a global sports injuries model with the goal of standardizing data related to the sports injuries subject.
\end{abstract}

Keywords: Health Informatics, Sports Injuries Model, Injuries Incidence and Prevalence, Socioeconomic Impact, Web Systems, Health Systems.

\section{Introduction}

Participation in sport and physical activity is considered a vital component of an active and healthy lifestyle, reducing the risk of various diseases and contributing to better social and physical performance [1]. However, as more population participates in such activities, we can consequently expect an increased number of sports injuries and simultaneously a substantial expense of financial healthcare resources and, possibly, absenteeism from work [2]. Actually, sports related injuries has been recognised as a major public health problem in many countries [3] and an accurate 
picture about incidence, prevalence and socioeconomics costs remains a challenge for all communities.

As far as we know, in Portugal there is not any consistent data about the number of sports related injuries at a recreational or even at different competitive levels, as well as, the costs related with amount of sports and working time lost. According to "The Burden of Sports Injuries in the European Union Report", the main objective is to assess the magnitude of sports injuries and their health and economic burden in all European Union countries. Additionally, this document suggests, as pre-requisite to such calculations, the development of a methodology for monitoring sport injuries based on widely available health statistics with the intention to provide data about frequency, severity, and cost of sport injuries as well as about its distribution over the different sports and population groups [4].

Motivated by the aforementioned, the project iReport SportsInjuries grew out of a partnership between the Informatics \& Systems Department and the Physiotherapy Department of the Polytechnic Institute of Setúbal. It was established that this project should consider two important aspects: i) the development of a sensitive sport injuries report interface, based on a global model, including the most important variables to characterize and assess sports injuries and socioeconomics costs, considering a standardized methodology and terminology based on literature recommendations [5] that would enable data comparison from different sports clinical contexts, sports populations or sports modalities [6]; ii) provide the data model and final reporting through an easy access system that allows users to register, collect, analyze and disseminate data through a quick, safe and effective method [7, 8]. It is also essential to have a flexible system, able to be upgraded or expanded according to the healthcare professionals and sports community usual and regular needs [7].

Regarding these fundamental requirements, we believe that iReport SportsInjuries would take advantage from a development into a Web platform. The Web application under development will allow health professionals to individually monitor and manage sports injuries information from sports organization, or even recreational athletes, and simultaneously contribute for an accurate picture at a national level. The project is further described in the following sections. In section 2, an overview of Web applications for sports injuries reports is presented. Section 3 presents the iReport SportsInjuries conceptual model that supports the information managed by the system, which is a global model to be followed by all organizations. Section 4 presents the developed prototype, which is a work in progress. We present conclusions and address further work in the last section.

\section{Web Applications for sport injuries reports}

In literature, we can find similar Web applications for management and monitoring of sports injuries. Most of these Web applications have been developed in the scope of 
research projects in order to obtain data required to calculate the injury incidence and prevalence levels $[9,10,11]$. Typically, these applications are composed of databases that aggregate injury data gathered from distinct fonts in order to provide a set of indicators [12]. Furthermore, some of these Web applications include athlete injury recovery software, allowing to register the injury and the treatment of each athlete. Some of this kind of software allows exporting data to main applications of monitoring and management sports injuries. As far as we know, the existing systems present a lack of information about the socioeconomics costs related to sports injuries.

NCAA Injury Surveillance System, actually supported by the Datalys Center, is one of the most cited sports injuries system in literature. This system was developed to support the NCAA Injury Surveillance Program [10], which maintains a program since 1982 for intercollegiate athletes. Recently, the system has been improved with an important engine that allows health professionals to export data from some known athletes training injury management software. On the other hand, Reporting Information Online [13] is a Web-based system developed at the Children's Hospital in Columbus, Ohio, conceived to register injuries on national bases. This software was one of the first systems allowing the registering of time loss due to the injury. One potential weakness reported on literature arises from the fact that this system was not integrated with NCAA Injury Surveillance System, resulting in potential duplication of registers in the USA.

Another example is the Fairfax County Public School Injury Surveillance Database [14]. This system was implemented to monitor sports injuries in the scope of sports activities in public high schools of Falls Church, in Virginia, USA. This system has proved to be robust enough to support more than a decade of use. However, it was conceived specifically to school sport, therefore it is not able to support the registration of injuries in a wider panorama that includes sports organizations and sports events. SIMS [15] is an advanced injury documentation and management software application designed to report athletes' participation status for training sessions or competition. It is a non-freeware software and it is conceived for the USA context. Another work is NExTT [16], an injury tracking software directed to athletic departments, which provides Web and mobile accesses directed to a set of features related to management of athletes' training sessions. This software also allows the management of athletes' data injuries as well as clinical history and training sessions. It has an engine to export data to some known surveillance systems.

Additionally, a common problem identified on almost all national injuries report systems is the data reliability [17]. Data reliability depends of several factors: (i) how concepts are understood by users that register the injury; (ii) how the existence of non-duplicate data or nonsense data is guarantee; (iii) how the access to change data is limited to authenticated users. Therefore, the design of a Web application software for sport injuries reports has to consider the implementation of mechanisms to guarantee that provided injuries information is reliable. None of the analyzed systems provide an easy and integrated way to individually support health professionals to 
report sports injuries at our national level. We believe that iReport SportsInjuries will represent a step forward for all Portuguese health professionals, and society at large, due to the current lack of information on sports injuries and their socioeconomics costs.

\section{3. iReport SportsInjuries' conceptual model}

Injury surveillance consists of an ongoing standardized collection of data describing the occurrence of, and risk factors associated with, an injury in large population groups. It provides the who, what, where, when, and how of information, which is the basis of descriptive epidemiology. Most of the times, data from injury registration is broad in scope, often missing uniformed detail, and sometimes lacking context [12]. This information needs to be tempered with a clinical mindset in order to be useful for distinct research approaches and to be used to answer important health related questions. Therefore, the design of the surveillance system's database is a crucial issue to the overall performance of the system.

We propose a global conceptual model for the sports injuries reports in order to guarantee that all data objects required by the database are completely and accurately represented and distinct stakeholders of the project can easily review and verify data. The UML Diagram that specifies the model is presented in Figure 1.
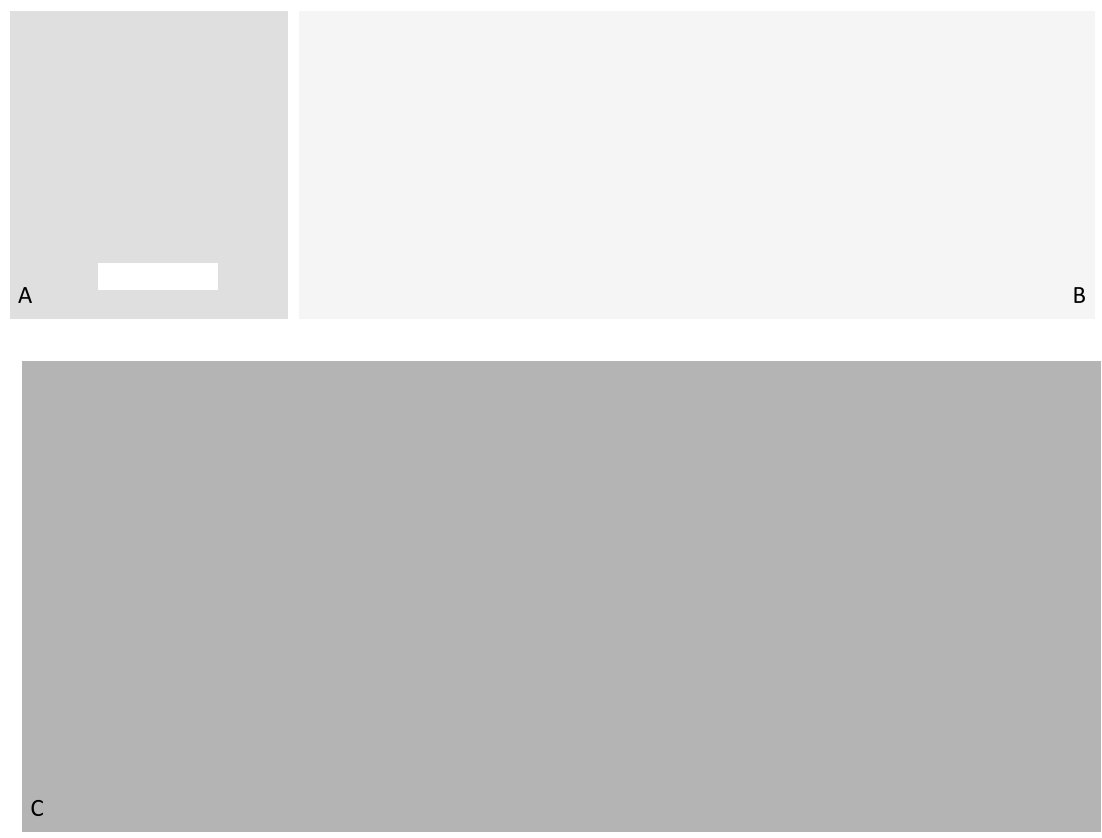

Figure 1 - UML Diagram of iReport SportsInjuries' Conceptual Model 
Three main groups of concepts can be identified in the proposed conceptual model:

- A - Injury Registration: Includes the injury data and the pos-injury data concepts. Both registers include the date of the occurrences. The dates are used to compute the period of time in which the athlete is inactive. The pos-injury register also includes information about the economics costs related to the injury.

- B - Injury Characterization: Defines the specification of the injury, which comprises the specification of the injured body parts, and for each body part is defined the structures affected (muscle, ligament, bone, etc.); for each structure is specified the respective injury mechanism (luxation, concussions, etc.), and the injury type.

- C - Stakeholders: This sub-model is responsible for the characterization of the main entities related to the injury: (i) the athlete that suffers the injury; for each athlete is also characterized the training schedule (hours per week, days per week, etc.) and the sport discipline. (ii) the health professional that registers the injury and treats the athlete. (iii) the organization in which the athlete is treated; in this case we can have two situations: the professional is associated to a sport organization, and in this case s/he follows a specific group of athletes that practice a specific sport discipline, or the professional treats the athlete in a health care unit without being associated to a specific sport organization.

\section{4. iReport SportsInjuries System}

The iReport SportsInjuries project has its origin in a previous project called SMLD, started in 2010 inside the Physiotherapy Department of our Institution. This project developed a survey to characterize injuries among Portuguese sports population. The survey was composed of two main sections: the sport injury registration and the socioeconomic impact registration. The first is composed of several dimensions including the athlete, the sport and the injury characterization. The second is centered on the injury socioeconomics consequences, such as the amount of sports, the working time spent and the monetary costs. The survey was implemented using the Google Docs platform whereas data was obtained through aggregated Excel facilities. The SMLD was validated and is being used in sports injuries prevention projects associated to football and futsal teams from Setúbal and Lisboa.

As mentioned before, the main goal of the iReport SportsInjuries project is to provide a secure, trustful and simple way to manage and analyze sports injuries data at a national level. In order to encourage injury data registration, a set of software services for injury reporting is provided to health professionals. These services will allow the health professionals to access anywhere, at any time, the injury history of their athletes, and additionally obtain a set of indicators about their athletes' injuries. In order to fulfill these features, the system has to support three types of users: the 
Health Professional (e.g., physiotherapists), the iReport Manager, and the Guest (which should apply to be accepted in the application as heath professional, waiting for the iReport Manager's approval), as documented in the UML user case diagram shown in Figure 2.

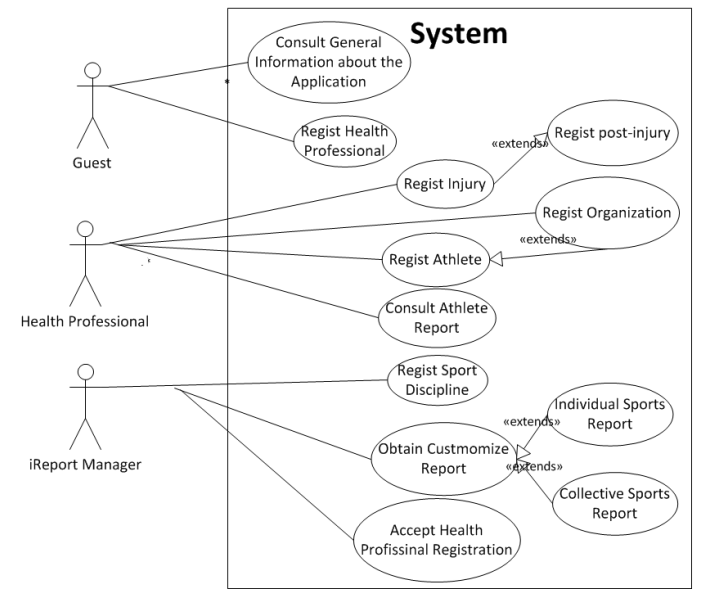

Figure 2 - iReport SportsInjuries System's User Case Diagram

The system supports the registration of a sport injury by a sport health professional (see Figure 3, left), where the registration comprises the two set of questions specified in the SMLD survey tool. The system guarantees the registration of information by authenticated users in order to fulfill data security requirements. Sport health professionals can consult the history of their athlete's injuries and obtain a set of indicators about injury incidence and prevalence (see Figure 3, right).

The iReport Manager profile gives access to unidentified data (the name of the athlete and his physiotherapist is encoded) about all the injuries registered on the system. The system also provides an analysis tool that allows project administrator to obtain customized reports (e.g., period of time, team sports discipline or an individual sport can be selected) on sports injuries incidence and sports injuries prevalence at a national level, since the system implements a global model that ends being followed by everyone, standardizing data. 
Figure 3 - iReport SportsInjuries Web Application' screens: (left) Injury Registration; (right) Report Injuries

The system was implemented following a thin-client server architecture. There is a central database based on SQL and the server logic implements the MVC pattern using Microsoft .net framework 4.

\section{Conclusions and Future Work}

The iReport SportsInjuries Web application is already available for a set of sports health professionals that are testing it. It is expected that this Web application satisfies the identified concrete needs concerning sports injuries information and their socioeconomics costs. This application will be available for free for all sports health professionals in Portugal. Therefore, it is expected that a massive use of the system will provide accurate data about sports injuries in diverse clinical contexts and sports organizations.

A mobile application for injury registration is planned to be developed. This new interface for mobile devices will allow sports health professionals to register injuries directly in their smartphones or tablets.

\section{References}

1. Beijsterveldt, A.M.C., Krist, et al.: Effectiveness and cost-effectiveness of an injury prevention programme for adult male amateur soccer players: design of a clusterrandomised controlled trial. Injury prevention 17 (2011).

2. Cumps, E., Verhagen, et al.: Injury rate and socioeconomic costs resulting from sports injuries in Flanders: data derived from sports insurance statistics 2003. British journal of sports medicine 42 (2008) 767-772.

3. Timpka, T., Lindqvist, et al: Impact of social standing on sports injury prevention in a WHO safe community: intervention outcome by household employment contract and type of sport. British journal of sports medicine 39 (2005) 453-457.

4. Kisser, R., Bauer, R.: The burden of sports injuries in the European Union. Research report D2h of the project Safety in Sports, Vienna, Austrian Road Safety Board (2012).

5. Finch, C.F., Ozanne-Smith, J., Williams, F.: The feasibility of improved data collection methodologies for sports injuries. Monash University Accident Research Centre (1995).

6. Meeuwisse, W.H., Love, E.J.: Athletic injury reporting. Sports Medicine 24 (1997) 184-204. 
7. Zemper, Dick: Epidemiology of Athletic Injuries.: ACSM's primary care sports medicine. Wolters Kluwer Health (2007).

8. Junge, A., Engebretsen, L., Alonso, J.M., RenstrÃIm, P., Mountjoy, M., Aubry, M., Dvorak, J.: Injury surveillance in multi-sport events: the International Olympic Committee approach. British journal of sports medicine 42 (2008) 413-421

9. Emery, C.A., Meeuwisse, W.H., Hartmann, S.E.: Evaluation of risk factors for injury in adolescent soccer implementation and validation of an injury surveillance system. The American journal of sports medicine 33 (2005) 1882-1891

10.Klossner, D., Corlette, J., Agel, J., Marshall, S.W.: Data driven decision making in practice: The NCAA injury surveillance system. New Directions for Institutional Research 2009 (2009) 53-63 11.

11.Fuller, C.W., Laborde, et al.: International rugby board rugby world cup 2007 injury surveillance study. British journal of sports medicine 42 (2008) 452-459 12.

12.Mechelen, W.v.: Sports injury surveillance systems. Sports Medicine 24 (1997) 164-168.

13.Darrow, C.J., Collins, C.L., Yard, E.E., Comstock, R.D.: Epidemiology of severe injuries among United States high school athletes 2005-2007. The American journal of sports medicine 37 (2009) 1798-1805.

14.Hinton, R.Y., et al. Epidemiology of Lacrosse Injuries in High Schoolar Aged Girls and Boys A 3-Year Prospective Study. The American journal of sports medicine 33 (2005) 1305-1314.

15.Lehr, M.E., Plisky, et al.: Field expedient screening and injury risk algorithm categories as predictors of noncontact lower extremity injury. Scandinavian journal of medicine \& science in sports (2013).

16.Solomon, D.J., et al., 2 Team Physicians Corner. 2012.

17.Thygerson, A.L., S.M. Thygerson, and J.S. Thygerson, Injury Prevention: Competencies for Unintentional Injury Prevention Professionals. Jones \& Bartlett Publishers (2008). 